\title{
The transcription factor IFN regulatory factor-4 controls experimental colitis in mice via T cell-derived IL-6
}

\author{
Jonas Mudter, ${ }^{1}$ Lioubov Amoussina, ${ }^{1,2}$ Mirjam Schenk, ${ }^{3}$ Jingling Yu, ${ }^{1,2}$ Anne Brüstle, ${ }^{4}$ \\ Benno Weigmann, ${ }^{2}$ Raja Atreya, ${ }^{2}$ Stefan Wirtz, ${ }^{2}$ Christoph Becker, ${ }^{2}$ Arthur Hoffman, ${ }^{1}$ \\ Imke Atreya, ${ }^{2}$ Stefan Biesterfeld, ${ }^{5}$ Peter R. Galle, ${ }^{1}$ Hans A. Lehr, ${ }^{6}$ Stefan Rose-John, ${ }^{7}$ \\ Christoph Mueller, ${ }^{3}$ Michael Lohoff, ${ }^{4}$ and Markus F. Neurath ${ }^{1,2}$ \\ 11st Medical Clinic and 2Institute of Molecular Medicine, University of Mainz, Mainz, Germany. \\ ${ }^{3}$ Institute of Pathology, University of Bern, Bern, Switzerland. ${ }^{4}$ Institute of Medical Microbiology, University of Marburg, Marburg, Germany. \\ ${ }^{5}$ Department of Pathology, University of Mainz, Mainz, Germany. ${ }^{6}$ Institut Universitaire de Pathologie, \\ Centre Hospitalier Universitaire Vaudois, Lausanne, Switzerland. ${ }^{7}$ Department of Biochemistry, University of Kiel, Kiel, Germany.
}

\begin{abstract}
The proinflammatory cytokine IL-6 seems to have an important role in the intestinal inflammation that characterizes inflammatory bowel diseases (IBDs) such as Crohn disease and ulcerative colitis. However, little is known about the molecular mechanisms regulating IL-6 production in IBD. Here, we assessed the role of the transcriptional regulator IFN regulatory factor-4 (IRF4) in this process. Patients with either Crohn disease or ulcerative colitis exhibited increased IRF4 expression in lamina propria $\mathrm{CD}^{+} \mathrm{T}$ cells as compared with control patients. Consistent with IRF4 having a regulatory function in T cells, in a mouse model of IBD whereby colitis is induced in RAG-deficient mice by transplantation with $\mathrm{CD}^{+} \mathrm{CD} 45 \mathrm{RB} \mathrm{B}^{\mathrm{hi}} \mathrm{T}$ cells, adoptive transfer of wild-type but not IRF4-deficient $T$ cells resulted in severe colitis. Furthermore, IRF4-deficient mice were protected from $T$ cell-dependent chronic intestinal inflammation in trinitrobenzene sulfonic acid- and oxazolone-induced colitis. In addition, IRF4-deficient mice with induced colitis had reduced mucosal IL-6 production, and IRF4 was required for IL-6 production by mucosal $\mathrm{CD}^{-} \mathrm{CO}^{+} \mathrm{T}$ cells, which it protected from apoptosis. Finally, the protective effect of IRF4 deficiency could be abrogated by systemic administration of either recombinant IL-6 or a combination of soluble IL-6 receptor (sIL-6R) plus IL-6 (hyper-IL-6). Taken together, our data identify IRF4 as a key regulator of mucosal IL- 6 production in T cell-dependent experimental colitis and suggest that IRF4 might provide a therapeutic target for IBDs.
\end{abstract}

\section{Introduction}

Inflammatory bowel diseases (IBDs) are characterized by chronic relapsing inflammations of the gastrointestinal tract that are not caused by specific pathogens. Based on recent studies, the pathogenesis of IBD seems to involve a complex interplay between certain genetic, environmental, and immunological factors. In particular, an unbalanced activation of the mucosal immune system driven by the commensal flora in a genetically susceptible host appears to cause intestinal inflammation in IBD patients (1-6). The activation of the mucosal immune system is characterized by production of proinflammatory cytokines (7-9). In particular, IL-6 and IL-6 signaling appear to play a pivotal role in IBDs. IL-6 is produced by both macrophages and T cells in IBD patients and mediates $\mathrm{T}$ cell resistance against apoptosis in chronic intestinal inflammation. The key functional role of IL-6 signaling was underlined by the observation that neutralizing anti-IL-6R antibodies led to suppression of established intestinal inflammation in animal models of IBD (10-13). Moreover, treatment of patients with Crohn disease (CD) with a humanized, neutralizing anti-IL-6R

Nonstandard abbreviations used: CD, Crohn disease; hyper-IL-6, sIL-6R plus IL-6; $\mathrm{HPF}$, high-power field; IBD, inflammatory bowel disease; IRF4, IFN regulatory factor-4; LP, lamina propria; LPMC, lamina propria mononuclear cell; sIL-6R, soluble IL-6 receptor; TNBS, trinitrobenzene sulfonic acid; UC, ulcerative colitis.

Conflict of interest: The authors have declared that no conflict of interest exists. Citation for this article: J. Clin. Invest. 118:2415-2426 (2008). doi:10.1172/JCI33227. antibody resulted in significantly higher response rates than placebo therapy, suggesting a therapeutic benefit of anti-IL- 6 signaling strategies (14). However, the molecular events that drive IL-6 production in IBD are poorly understood.

The IFN regulatory factor-4 (IRF4, also called PIP, LSIRF, and ICSAT) is a member of the IRF family of transcriptional regulators (15-19). It was originally discovered as a new IRF protein that acts as a binding factor to the Ig light chain enhancer in association with the transcription factor PU.1. IRF4 is expressed in B cells, mature $\mathrm{T}$ cells, dendritic cells, and macrophages, and its expression is upregulated by IgM or TCR cross-linking as well as costimulation. As the expression of IRF4 is primarily confined to lymphocytes, it has been proposed that IRF4 controls lymphocyte responses. Indeed, IRF4 together with NFATc2 has been shown to induce IL-4 gene transcription (20). In addition, studies in IRF4-deficient mice have shown profound defects in mature B and T cell function. Subsequent in vivo studies of IRF4 function identified its critical role in helper T cell differentiation (16): in Leishmania major infection, lymph node cells from $\mathrm{IRF4}^{+/-}$mice showed significantly higher IL-4 and IFN- $\gamma$ secretion than cells from $I R F 4^{-/-}$mice. Furthermore, in vitro differentiation studies showed strongly impaired Th1 and Th2 cytokine responses of $\mathrm{IRF}^{-/-} \mathrm{T}$ cells. The defect in IL-4-dependent Th2 differentiation was due to the inability of $I R F 4^{-/-}$lymphocytes to upregulate expression of the master transcription factor GATA-3. In turn, retroviral overexpression of GATA-3 restored the capacity to undergo Th2 differentiation. 

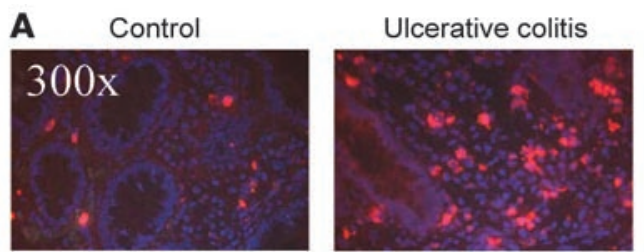

C

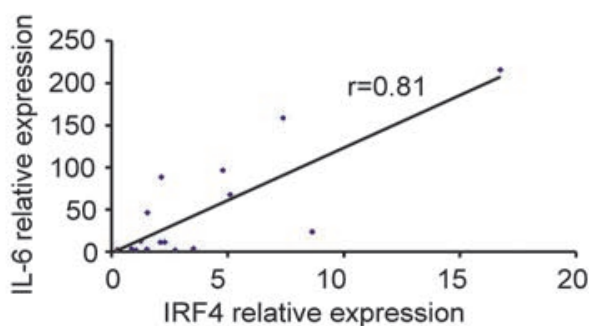

E

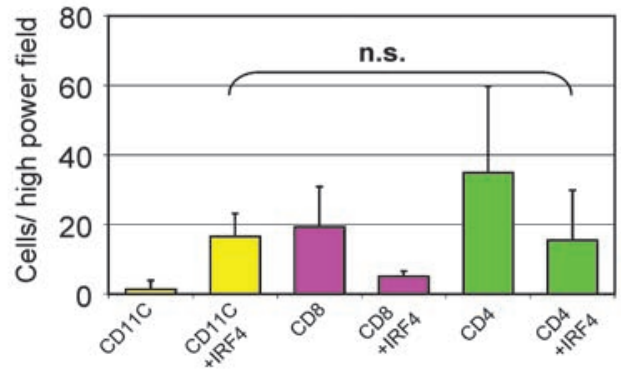

Crohn disease

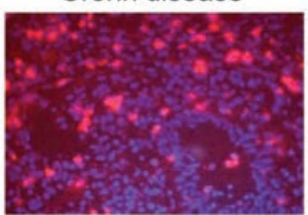

D
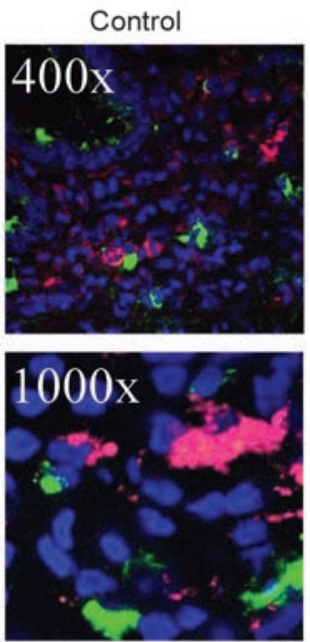

B
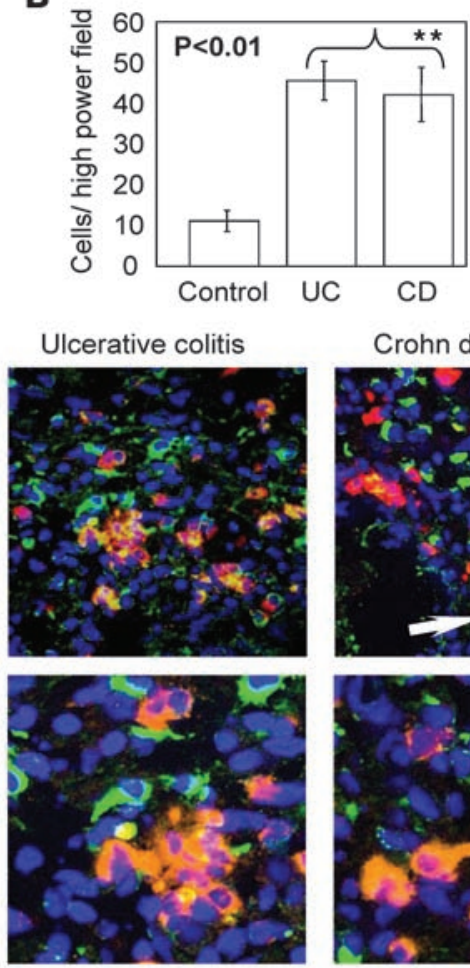

Crohn disease
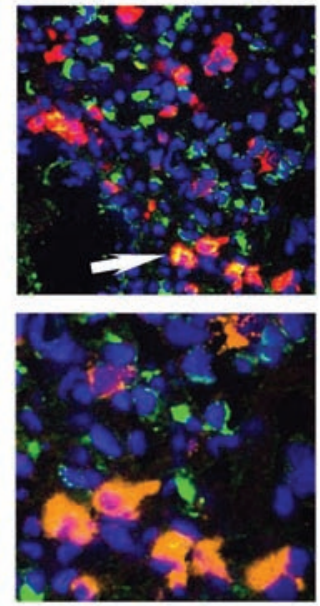

\section{Figure 1}

IRF4 expression is increased in inflammatory bowel diseases. (A) Immunofluorescence staining of human mucosal cryosections for IRF4. Cryosections of gut specimens from control patients and IBD patients ( $n=10$ per group) were stained with antibodies against IRF4 (red). Nuclei were counterstained with DAPI (blue). Original magnification, $\times 300$. (B) Quantitative assessment of IRF4-positive cells. IRF4-positive cells were counted in 7 HPFs per patient. There was a significantly increased number of IRF4-expressing cells in both CD and UC patients as compared with control patients. ${ }^{* *} P<0.01$. (C) Quantitative assessment of IRF4 and IL-6 mRNA in the lamina propria. IRF4 and IL-6 mRNA levels in mucosal biopsies were determined by quantitative PCR. A total of 16 IBD patients were analyzed. Values for IRF4 and IL- 6 were strongly correlated $(r=0.81)$ in IBD. (D) Immunofluorescence double staining for IRF4 and CD3. Double-staining analysis was performed (green, IRF4-positive cells; red, CD3-positive cells). Original magnification, $\times 400$ (upper panels); $\times 1000$ (lower panels). Cells coexpressing IRF4 and CD3 appeared yellow (arrow). Staining analysis revealed that a large number of IRF4-positive cells coexpress CD3 on their surface. (E) Immunofluorescence double staining for IRF4 and CD4, CD8, and CD11c. Cells were stained using anti-IRF4 antibodies, and additional surface staining was performed using antibodies against either CD11C, CD8, or CD4. The number of positive cells was analyzed in 7 HPFs per patient $(n=6)$. CD11c, CD8, and CD4 single-positive cells and the number of double-positive cells were counted. Data represent mean values \pm SD.

Based on the above data, we have analyzed in the present study the role of IRF4 in experimental colitis in mice. We found that IRF4 plays a key regulatory role for IL-6 production and consecutive activation of T lymphocytes in experimental colitis.

\section{Results}

Increased expression of IRF4 in mucosal T cells in IBD. In an initial series of studies, we aimed at analyzing the expression of IRF4 in the mucosa of patients with IBD. To assess expression of IRF4 in CD and ulcerative colitis (UC), we used colonic mucosa derived from surgical resections. Cryosections from colonic samples were analyzed for IRF4 expression by immunofluorescence staining. As shown in Figure 1A, many IRF4-positive mononuclear cells were detected in the lamina propria of IBD patients, whereas relatively few IRF4-positive cells were found in control patients. Quantitative assessment showed a significantly increased number of IRF4expressing cells in both CD and UC as compared with controls (Figure 1B). Furthermore, IRF4 mRNA expression in the mucosa of IBD patients correlated well with mucosal IL-6 mRNA levels $(r=0.81)$ (Figure 1C). Although this finding does not directly prove that IRF4 regulates IL- 6 gene transcription in human lamina propria T cells, our results thus suggested that IRF4 might play a role in regulating mucosal inflammation in IBD.

As IRF4 is expressed in APCs and T cells, we next determined whether CD3-positive lamina propria T cells in IBD would express IRF4. Indeed, we found by double-staining analysis that a large number of CD3-bearing cells were also positive for IRF4 (Figure 1D), consistent with a potential regulatory role of IRF4 in mucosal T lymphocytes in IBD. Furthermore, double-staining analysis revealed that many CD11 $c^{+}$cells express IRF4 (Figure 1E). To identify $T$ cell subsets expressing IRF4, we subsequently performed double-staining analysis for CD4 or CD8 and IRF4 (Figure 1E). The number of mucosal CD4/IRF4 double-positive cells was on average about 2-fold higher as compared with CD8/IRF4 doublepositive cells, suggesting that the majority of $\mathrm{T}$ cells expressing IRF4 in IBD are positive for CD4.

Attenuation of inducible, $T$ cell-dependent experimental colitis in IRF4-knockout mice. To determine the functional role of IRF4 in 
A
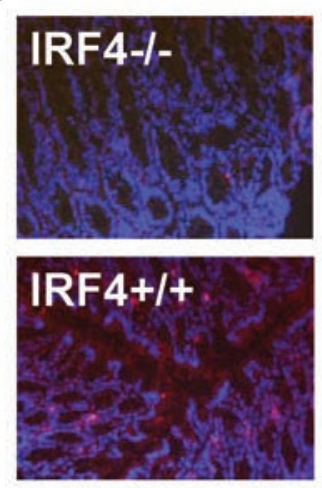

IRF4+/+ Oxa

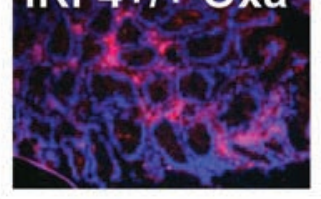

E

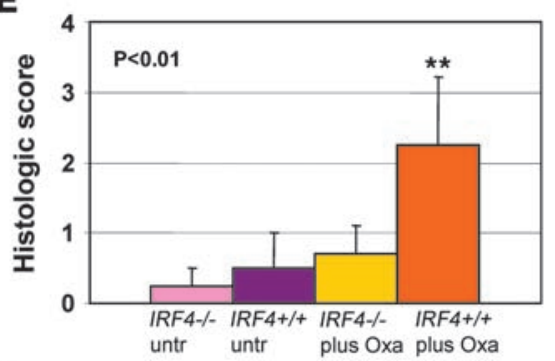

G

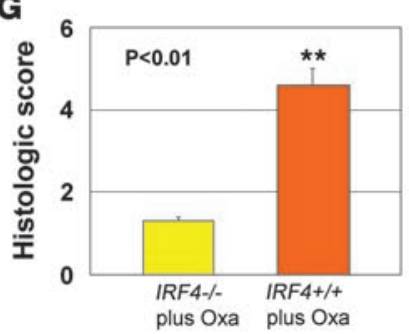

B
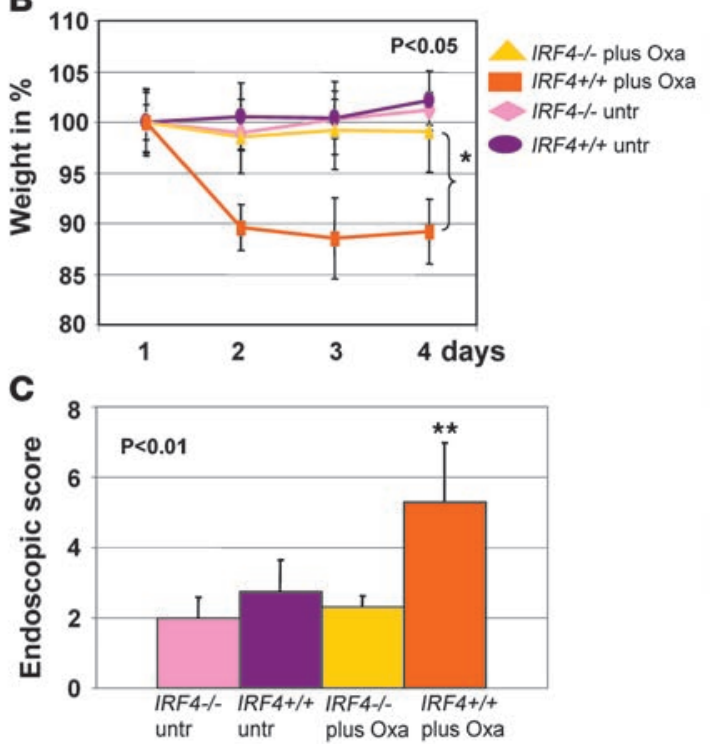

$\mathbf{F}$
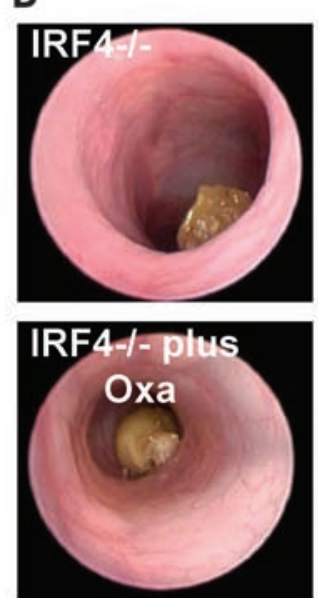

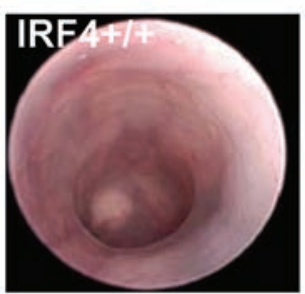

IRF4+/+ plus Oxa

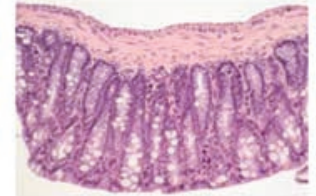

IRF4-/- plus Oxa

H

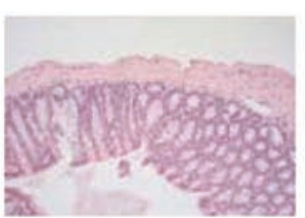

IRF4-/- plus Oxa

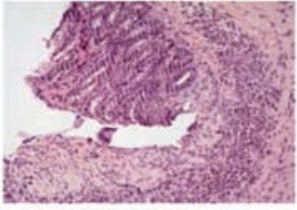

IRF4+/+ plus Oxa

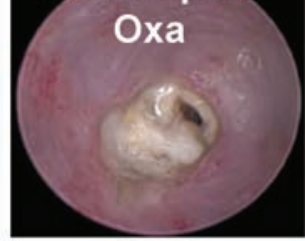

Figure 2

Abrogation of oxazolone-induced colitis in IRF4-deficient mice. (A) IRF4 expression was analyzed by immunofluorescence (red) in oxazolone colitis. IRF4-/- mice served as negative control (upper panel). Colitic WT mice had many IRF4-positive cells in the lamina propria (lower panel) as compared with untreated WT mice (middle panel). Original magnification, $\times 400 .{ }^{*} P<0.05$. (B) WT $(n=8)$ and IRF4-deficient mice $(n=11)$ were treated by rectal administration of oxazolone following prior sensitization. WT $\left(I R F 4^{+/+}\right)$mice lost significantly more weight as compared with oxazolone-treated IRF4-/- mice, untreated WT mice $(n=5)$, or untreated knockout mice $(n=3)$. ${ }^{\star \star} P<0.01$ on days 3 and 4 . (C) IRF4-deficient mice showed a significantly reduced endoscopic score. (D) Representative endoscopic pictures from each group are shown. (E) Histological scoring revealed a significantly higher degree of inflammation in oxazolone-treated WT mice as compared with $I R F 4^{-/-}$mice. Data are given as mean values \pm SEM (IRF4+/+ plus oxazolone, $n=8$; IRF4 $4^{-/-}$plus oxazolone, $\left.n=11 ; I R F 4^{+/+}, n=5 ; I R F 4^{-/-}, n=3\right)$. (F) Histological findings

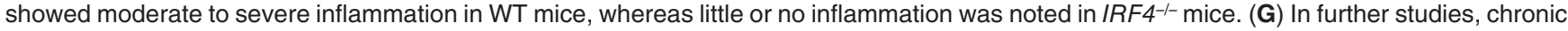
oxazolone colitis was evaluated. Mice were treated 3 times by intrarectal application of oxazolone and evaluated on day 21. A more severe colitis activity was induced in WT mice $(n=5)$ as compared with $I R F 4^{-/-}$mice $(n=5)(\mathbf{F})$. This was underlined by differences $\left({ }^{* *} P<0.01\right)$ in the histologic score between both groups (G). (H) Representative histologic pictures from each group are shown. Original magnification, $\times 100$.

gut inflammation, we took advantage of IRF4-deficient mice (15). In these studies, we subjected IRF4-deficient mice and WT control mice to $\mathrm{T}$ cell-dependent, inducible models of experimental colitis caused by hapten reagents (oxazolone, trinitrobenzene sulfonic acid [TNBS]) that exhibit some similarities to $\mathrm{CD}$ and UC in humans.
IRF4-expressing cells were increased in the mucosa of WT mice upon oxazolone treatment as compared with untreated mice (Figure 2A). The functional relevance of this observation was underlined by the observation that IRF4-knockout mice were completely protected from oxazolone-induced colitis, whereas WT mice showed marked diarrhea and weight loss (Figure 2B). 
A

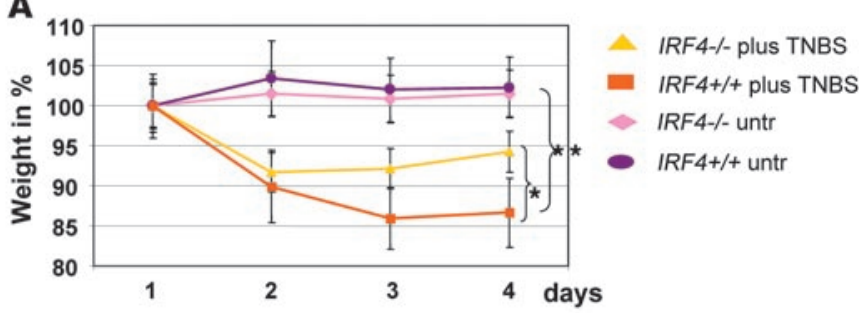

C
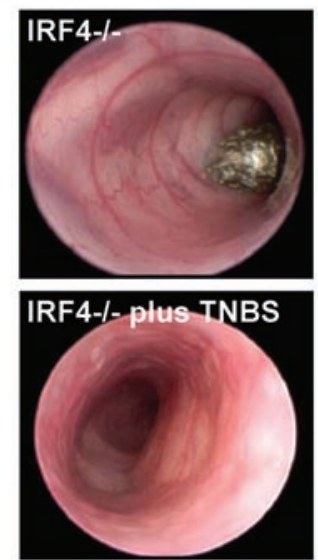

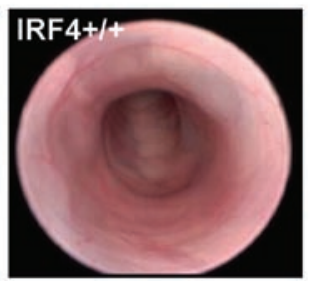

IRF4+1+ plus TNBS

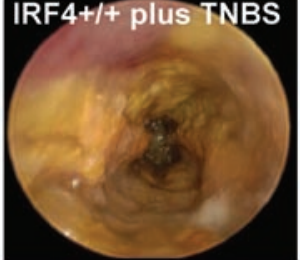

B

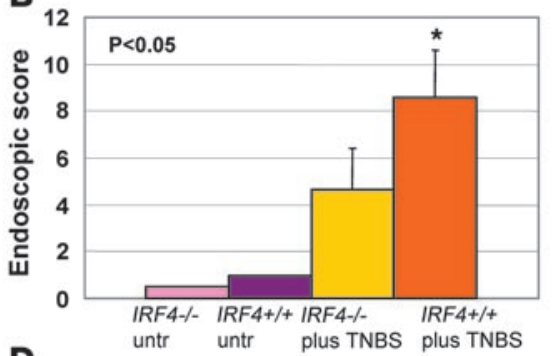

D

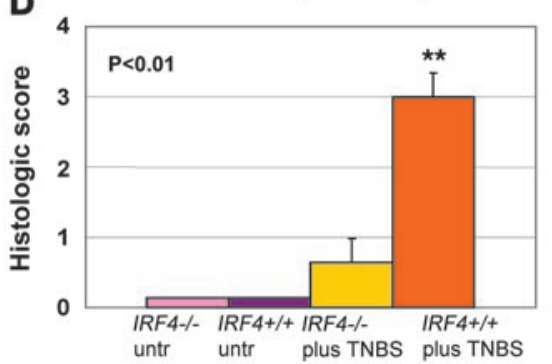

E

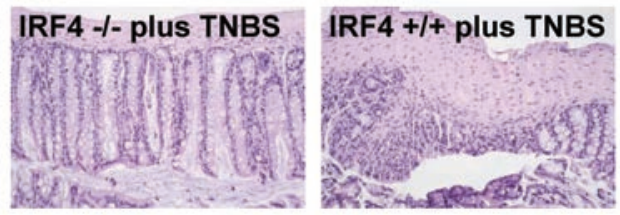

Figure 3

Attenuation of TNBS-induced colitis in IRF4-deficient mice. (A) IRF4-deficient mice and WT control mice were treated by intrarectal administration of the hapten reagent TNBS followed by monitoring of body weight. TNBS-treated IRF4- mice lost significantly $(P<0.05)$ less weight as compared with WT mice. One representative experiment out of 3 is shown. Endoscopic (B and C) and histologic (D and E) analyses showed significantly reduced inflammation in TNBS-treated IRF4-/- mice as compared with TNBS-treated WT mice. ${ }^{*} P<0.05$; ${ }^{* *} P<0.01$. Data are given as mean values \pm SEM (IRF4+/+ plus TNBS, $n=17$; IRF4 $4^{-/}$plus TNBS, $n=14 ; I R F 4^{+/+}, n=8$; IRF4 $\left.4^{-/-}, n=11\right)$. Data were pooled from 3 independent experiments. Original magnification, $\times 100$.

In addition, we performed endoscopic analysis of colitis severity (Figure 2, C and D). Whereas the colonic mucosa of IRF4-/- mice was unaffected, WT mice showed severe inflammation of the mucosa. Consistently, histologic assessment of colitis showed a significantly higher inflammation score in WT mice than in IRF4-deficient mice, and the mean score in the latter mice was similar to that in healthy, untreated control mice (Figure 2, E and F). To assess the role of IRF4 in a more intense form of oxazolone colitis, WT and IRF4-deficient mice were sensitized to oxazolone followed by intrarectal application of oxazolone after 7,12 , and 17 days and final analysis after 21 days. A severe colitis with erosions and ulcer formations was noted in WT mice, whereas IRF4-deficient mice were almost completely protected from colitis. Consistently, histologic scoring revealed a significantly higher colitis activity in WT mice as compared with IRF4deficient mice (Figure 2, G and $\mathrm{H}$ ).

In subsequent experiments, we challenged WT and IRF4knockout mice with the hapten reagent TNBS. Interestingly, $I R 4^{-/-}$mice lost less body weight than WT mice upon TNBS administration (Figure 3A). Furthermore, the former had significantly attenuated colitis activity as compared with the latter mice by endoscopic scoring (Figure 3, B and C). In fact, high resolution mini-endoscopy revealed severe inflammation with ulcers and contact bleeding in WT mice, whereas TNBS-treated $I R F 4^{-/-}$mice exhibited only mild to moderate signs of inflam- mation (Figure 3C). Consistently, IRF4-knockout mice showed a significant reduction of the histologic colitis score as compared with WT mice (Figure 3, D and E).

IRF4 controls IL-6 production in T cell-dependent experimental colitis. To characterize the mechanisms for the observed differences in inflammatory activity between WT and IRF4-deficient mice, we next analyzed cytokine expression. Interestingly, whereas equal mRNA levels of the proinflammatory cytokine TNF were detected in the colons of WT and knockout mice, IL- 6 mRNA levels in the lamina propria were higher in oxazolone-treated WT mice as compared with oxazolone-treated $I R F 4^{-/-}$mice (Figure 4A). In TNBStreated $I R F 4^{-/-}$mice, IL-6 mRNA was also strikingly decreased compared with WT mice. In contrast, levels of the proinflammatory cytokine TNF were similar in both groups (Figure 4B). IFN- $\gamma$ production was reduced on average in the absence of IRF4, although this was not statistically significant (Figure 4B).

As T cells and APCs can produce IL-6, we next determined the ability of lamina propria mononuclear cells (LPMCs) from untreated mice to respond to $\mathrm{T}$ cell-specific stimulation (Figure 5A). Upon $\mathrm{T}$ cell-specific stimulation with anti-CD3/CD28 antibodies, IRF4-/LPMCs produced significantly lower amounts of IL-6 protein as compared with WT LPMCs. In contrast, under unstimulated conditions, IL-6 production was comparable between $\mathrm{IRF}^{-{ }^{--}}$and WT LPMCs (Figure 5A), suggesting that T cell-dependent IL-6 production is reduced in the absence of IRF4. Indeed, IL- 6 production 
A
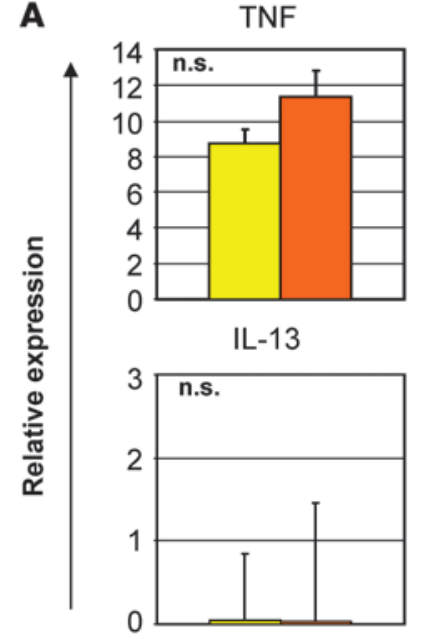

B

들

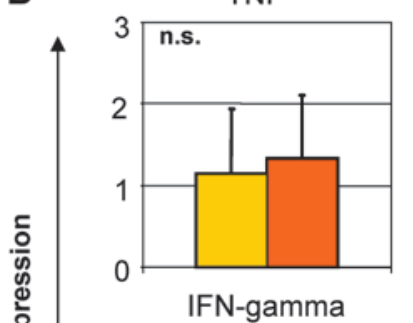

IFN-gamma

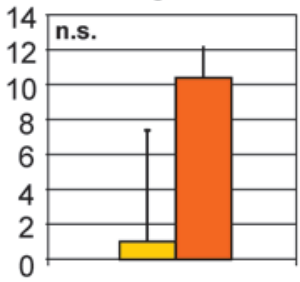

TGF-beta

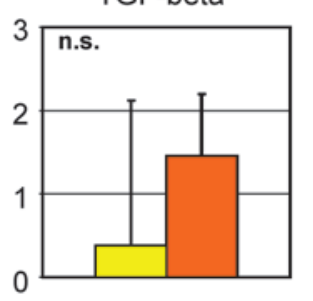

口IRF4-/- Oxa

口 IRF4+/+ Oxa
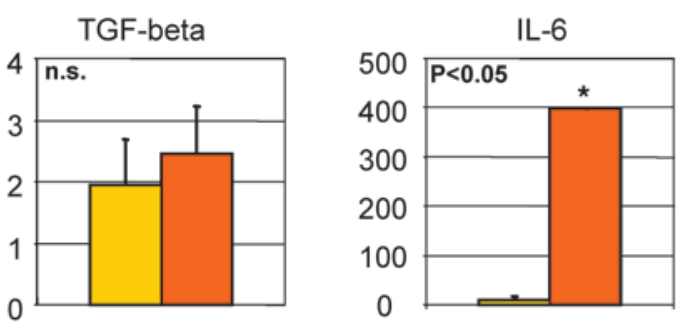

\section{Figure 4}

Differences in mucosal IL-6 expression between WT and IRF4-deficient mice. On day 4 , colons of the different groups of mice were analyzed for cytokine levels by quantitative PCR. Relative expression levels of IL-6, TNF, TGF- $\beta$, IFN- $\gamma$, and IL-13 mRNA were measured by quantitative real-time RT-PCR. Values were normalized to $\beta$-actin expression levels. (A) The mRNA expression of various pro- and antiinflammatory cytokines in the colons of oxazolone-treated IRF4-deficient and WT mice was analyzed by quantitative PCR ( $n=8$ per group). Whereas no differences were noted in the expression of TGF- $\beta$ and TNF, IL- 6 mRNA expression was strongly induced in WT but not IRF4-deficient mice upon oxazolone administration. In addition, there were no significant differences in IL-13 mRNA levels between the groups. ${ }^{* \star} P<0.01$. (B) The mRNA expression pattern of several cytokines was also determined in TNBS colitis ( $n=8$ per group). Whereas no significant differences in the expression of the proinflammatory cytokines TNF and IFN- $\gamma$ were noted between the groups, IL-6 mRNA expression was strongly induced in WT but not IRF4-deficient mice upon TNBS administration. ${ }^{*} P<0.05$. by $\mathrm{T}$ cell-enriched LPMCs and purified $\mathrm{CD} 90^{+}$lamina propria $\mathrm{T}$ cells from TNBS-treated IRF4-deficient mice was significantly reduced as compared with WT mice (Figure 5, B and C). However, IL-6 production by splenic T cells was not different between WT and knockout cells (Figure 5D), suggesting that mucosal rather than systemic IL-6 levels are controlled by IRF4. Consistently, the number of IL-6-expressing cells in the lamina propria of TNBStreated IRF4-deficient mice was significantly lower than in control mice (Figure 5, E and F).

As mucosal IL-6 production is a key regulator of T cell resistance against apoptosis in colitis (15), we next determined whether the reduced mucosal IL-6 levels in IRF4-/- mice would affect apoptosis of lamina propria cells in experimental colitis (Figure 5G). Interestingly, more apoptotic mononuclear cells were found in the mucosa of IRF4-knockout mice as compared with WT controls, suggesting that IRF4 regulates mucosal IL-6 levels and T cell apoptosis in TNBS colitis. Indeed, double-staining analysis revealed that apoptosis was induced in CD3-positive lamina propria $\mathrm{T}$ cells from $I R 4^{-/-}$but not WT mice (Figure $5 \mathrm{H}$ ).

The protective phenotype of IRF4 ${ }^{-/-}$mice is abrogated by administration of hyper-IL-6. To assess whether the reduced mucosal IL- 6 production in $\mathrm{IRF}^{-{ }^{--}}$mice was relevant in vivo, we next injected recombinant IL- 6 or the designer protein hyper-IL-6 (IL-6 plus soluble IL-6 receptor: IL-6/sIL-6R) intraperitoneally into TNBS-treated
IRF4-knockout mice (Figure 6A). While such treatment had little or no effects in WT mice, both treatment modalities abrogated the protective phenotype of IRF4-deficient mice in TNBS colitis, and such mice developed severe colitis with weight loss and mucosal ulcerations as well as histologic evidence of colitis (Supplemental Figure 1; available online with this article; doi:10.1172/ JCI33227DS1). As these data suggested that IL-6 signaling is important to suppressing the protective effects caused by IRF4 deficiency, we next tested the effects of hyper-IL-6 in oxazolonetreated IRF4 ${ }^{-/-}$mice (Figure 6B). Administration of hyper-IL-6 completely blocked the protective effect of $I R F 4^{-/-}$deficiency in oxazolone-induced colitis, and colitis activity was comparable between oxazolone-treated WT mice and hyper-IL-6-treated IRF4-knockout mice (Figure 6B). In fact, endoscopic images and histologic analysis showed severe mucosal inflammation upon hyper-IL-6 administration (Supplemental Figure 1).

We next evaluated cytokine patterns in hyper-IL-6-treated mice to clarify proinflammatory mechanisms inducing colitis in the otherwise protected IRF4 phenotype (Figure 7A). Importantly, hyper-IL-6 treatment significantly induced mucosal IL-6 mRNA levels in TNBS-treated IRF4-deficient mice (14-fold induction), although levels were lower than in TNBS-treated WT mice. In contrast, no significant changes in expression levels of TNF and TGF- $\beta$ were noted (Figure 7A), suggesting that IRF4 is a key regu- 

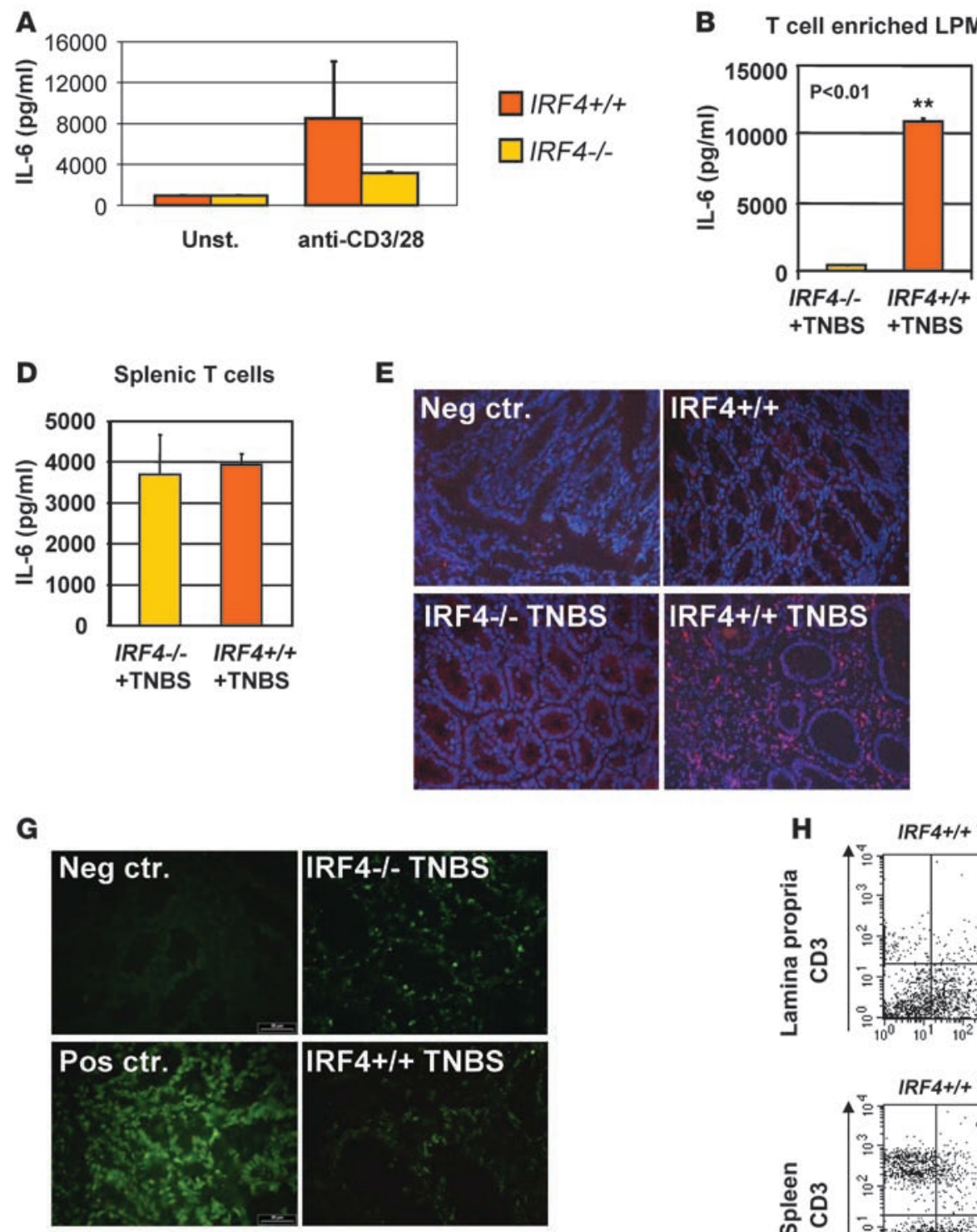
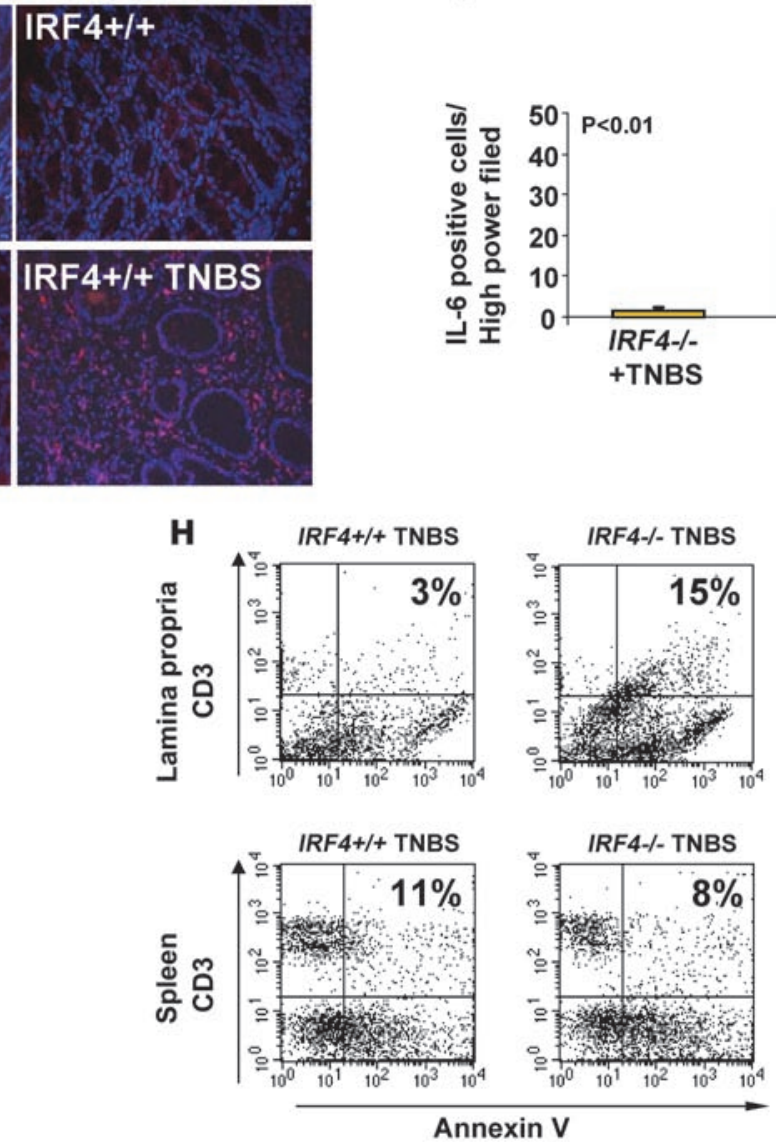

Figure 5

Decreased activation of the antiapoptotic IL-6 pathway in IRF4-deficient T cells. (A) LPMCs from WT and IRF4-deficient mice were cultured with or without anti-CD3/CD28 antibodies. IL-6 production was higher in WT mice as compared with IRF4-deficient mice upon costimulation. (B-D) T cell-enriched LPMCs (B), CD90+ LP T cells (C), or splenic T cells (D) were isolated from TNBS-treated mice and stimulated for 2 days. LP cells but not spleen cells from TNBS-treated IRF4-deficient mice produced significantly lower amounts of IL-6 than cells from TNBS-treated WT mice ( $n=9-10$ mice per group; $\left.{ }^{\star \star} P<0.01\right)(E)$ TNBS-treated WT mice exhibited an increased number of IL-6-positive cells (red) in the lamina propria as compared with controls. Original magnification, $\times 300$. (F) IL-6-positive cells were counted in TNBS-treated IRF4 ${ }^{-/-}$and WT mice $(5 \mathrm{HPFs})$. IL-6-positive cells were increased in TNBS-treated WT mice compared with IRF4-deficient mice. (G) Colonic cryosections were stained using TUNEL assays. There were more positive cells in the colons of TNBS-treated IRF4-knockout mice than in TNBS-treated WT mice $(n=6$ per group). Positive and negative control samples are shown. Original magnification, $\times 300$. (H) Lamina propria cells were isolated from either TNBS-

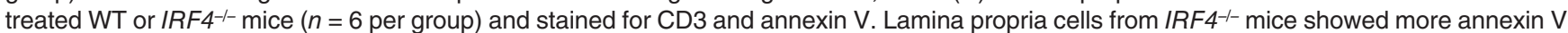
and CD3 double-positive cells than those from WT mice (15\% versus $3 \%)$. No such differences were detected in spleen cells ( $8 \%$ versus $11 \%$ ).

lator of mucosal IL-6 levels. In addition, induction of IL-6 levels upon hyper-IL-6 administration in IRF4-deficient mice was associated with a suppression of mucosal mononuclear cell apoptosis (Figure 7, B-D), indicating that such treatment prevents apoptosis in experimental colitis.
The protective effect of IRF4 deficiency is mediated via T cells. As the above colitis models are mediated by T cells, we next tested the possibility that the protective effect of IRF4 deficiency in experimental colitis is mediated via T cell-derived IL-6. Accordingly, we chose to determine the effects of IRF4 in a transfer colitis model in which 
A
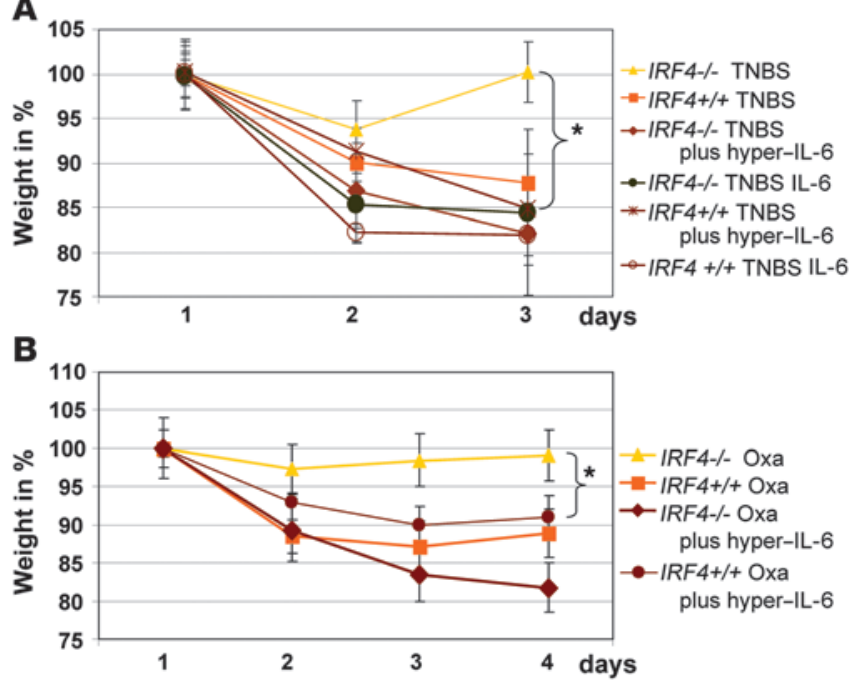

Figure 6

The protective effect of IRF4 deficiency is abrogated in TNBS and oxazolone colitis by administration of recombinant IL-6 or hyper-IL-6. (A) To determine the functional role of IL-6 signaling for the effects of IRF4 on TNBS colitis activity in vivo, recombinant IL-6 or the designer fusion protein hyper-IL-6 was intraperitoneally administered to TNBStreated IRF4-deficient mice. TNBS-treated IRF4-/- mice exhibited severe inflammation and weight loss upon IL-6 or hyper-IL-6 treatment that was indistinguishable from colitis in WT control mice (TNBS-

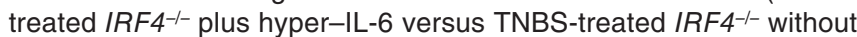
hyper-IL-6; $\left.{ }^{*} P<0.05\right)$. Data are given as mean values \pm SEM $\left(I R F 4^{+/+}\right.$

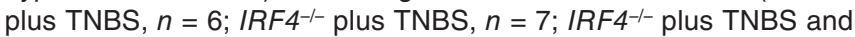

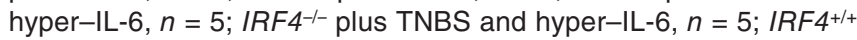
plus TNBS and IL-6, $n=4$; IRF4-/- plus TNBS and hyper-IL-6, $n=5$, in 2 independent experiments). (B) To determine the functional role of IL-6 signaling for the effects of IRF4 on oxazolone colitis activity in vivo, hyper-IL-6 was intraperitoneally administered to oxazolonetreated IRF4-deficient mice. Oxazolone-treated IRF4-/- mice exhibited severe inflammation and weight loss upon hyper-IL-6 treatment that was indistinguishable from colitis in WT control mice (oxazolone-treat-

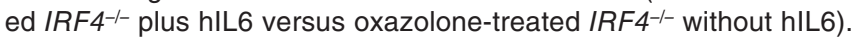
Data are given as mean values \pm SEM (IRF4 $4^{+/+}$plus oxazolone [oxa], $n=12$; IRF4 $4^{-/}$plus oxazolone, $n=13$; IRF4-/- plus oxazolone plus hyper-IL-6, $n=7$; IRF4+/+ plus oxazolone plus hyper-IL-6, $n=6$, in 3 independent experiments).

$\mathrm{CD} 4^{+} \mathrm{CD} 45 \mathrm{RB}^{\text {hi }} \mathrm{T}$ cells are adoptively transferred into immunocompromised hosts. Splenic CD45RB hi $\mathrm{CD} 4^{+}$cells were isolated either from WT or IRF4-/ mice. Cells were then intraperitoneally injected in immunodeficient RAG mice. Within 15 days, RAG mice reconstituted with $\mathrm{CD} 45 \mathrm{RB}^{\text {hi }} \mathrm{CD} 4^{+} \mathrm{T}$ cells from WT mice showed diarrhea and lost body weight (Figure 8A). In contrast, RAG mice reconstituted with $\mathrm{CD} 45 \mathrm{RB}^{\text {hi }} \mathrm{CD} 4^{+} \mathrm{T}$ cells from $I R F 4^{-/}$mice gained weight (Figure 8A). Consistently, colonoscopy of mice in the latter group revealed no signs of mucosal inflammation, whereas RAG-deficient mice reconstituted with WT CD45RB ${ }^{\text {hi }} \mathrm{CD} 4^{+} \mathrm{T}$ cells exhibited severe mucosal inflammation (Figure 8, B and D). Moreover, the latter mice showed a significantly higher histologic score of colitis activity than the former mice (Figure 8C).

Reduced IL-6 production in RAG-knockout mice transferred with IRF4-/$T$ cells. We next analyzed cytokine expression in RAG-recipient mice reconstituted with $\mathrm{CD} 45 \mathrm{RB}^{\text {hi }} \mathrm{CD} 4^{+}$cells from WT or $I R F 4^{-/}$mice. Whereas the mRNA expression of IL- 6 was significantly reduced in the latter as compared with the former mice, little or no significant changes in the expression of TNF and TGF- $\beta$ were noted (Figure $9 \mathrm{~A})$. Furthermore, there was a higher induction of mucosal mononuclear cell apoptosis in RAG-knockout mice reconstituted with IRF4-deficient cells as compared with RAG-knockout mice given WT cells (Figure 9B), consistent with the idea that IRF4 regulates survival of mucosal T cells.

\section{Discussion}

IRF4 is a lymphoid- and myeloid-restricted member of the IRF family of transcription factors that binds to IFN sequence response elements present within the promoters of IRF-regulated genes (15, $18,21)$. In the present study, we have identified a key regulatory role of IRF4 in controlling IL- 6 production by mucosal T cells and subsequently T cell apoptosis. Studies in 3 different models of chronic intestinal inflammation revealed that IRF4 plays a pathogenic role in colitis by regulating mucosal IL- 6 production. Importantly, administration of IL-6 or hyper-IL-6 prevented mucosal cell apoptosis and abrogated the protective effect of IRF4 deficiency, suggesting that IRF 4 exerts its effects by modulating IL-6 production. These data provide what we believe are novel insights into the pathogenesis of IBDs and suggest that targeting of IRF4 may be beneficial for therapy of intestinal inflammation.

Although an important role of IRF4 in T cell development has been described $(15,16,19,20)$, its regulatory role in chronic intestinal inflammation was poorly understood. Here, we have analyzed the role of IRF4 in intestinal inflammation using IRF4-deficient mice. Our data reveal that IRF4 plays an important role in several T cell-mediated models of experimental colitis. Specifically, IRF4 deficiency suppressed both oxazolone- and TNBS-induced colitis. Furthermore, we addressed the role of IRF4 in T cells, as recent data have shown that IRF4 is also expressed in B cells and APCs (22-24). Interestingly, we found that IRF4-deficient T cells fail to induce colitis in adoptive transfer experiments in RAG-knockout mice, suggesting that the protective capacity of IRF4 deficiency is at least partially due to effects in $T$ lymphocytes.

In further mechanistic studies, we analyzed the cytokine production in experimental colitis. In TNBS-induced colitis, we found that IL-6 expression was strongly upregulated in the inflamed colon of WT but not IRF4-deficient mice. Interestingly, IL-6 production of splenic cells in colitis was not different between the WT and $I R F 4^{-/}$groups, suggesting that IRF4 regulates mucosal rather than systemic IL-6 levels in experimental colitis. Moreover, we recently found that, similarly to peripheral T cells (25), the mucosal production of IL-17 was strikingly reduced in the absence of IRF4, possibly at least partially due to the reduced expression of the IL-6 inducible transcription factor RORgammat (J. Mudter et al., unpublished observations). The latter transcription factor plays a fundamental role in the development of Th17 cells, a T cell subset that can be induced by stimulation of T cells with IL-6 plus TGF- $\beta$ and activated with IL-23 (26-29). Thus, our data suggest a model in which IRF4 regulates mucosal IL-6 production, RORgammat levels, and Th17 development. However, IL-17A-deficient mice develop normal TNBS and oxazolone-induced colitis, and $\mathrm{T}$ cells derived from such mice are fully capable of inducing colitis in reconstituted RAG-knockout mice (B. Weigmann et al., unpublished observations). Thus, factors other than IL-17A play an important role in mediating the effects of IRF4 in T cell-mediated colitis. As Th17 cells are known to produce IL-17F and IL-22 in addition to IL-17A, it is possible that these cytokines rather 
A

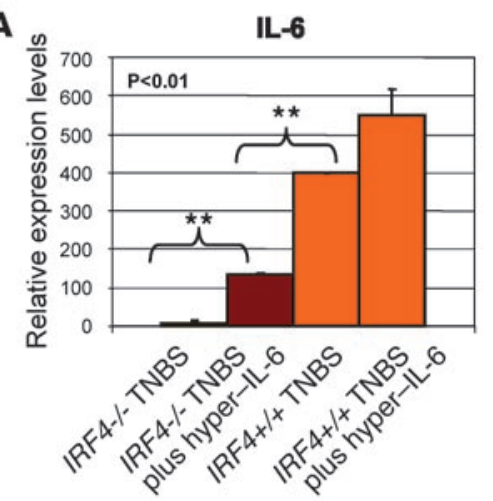

B

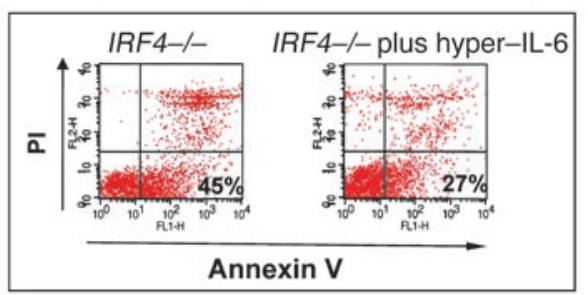

D
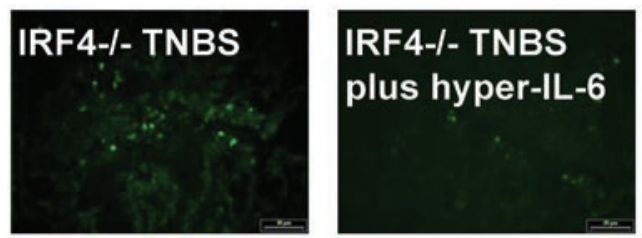

TNF

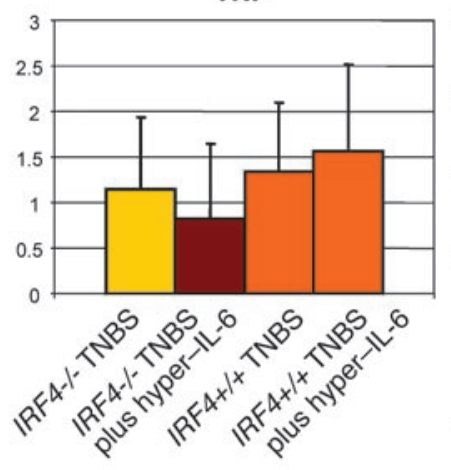

TGF-beta

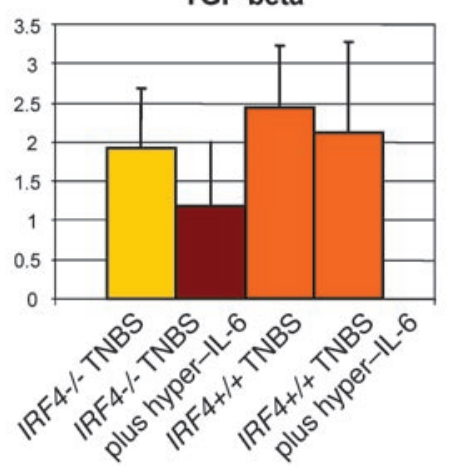

C

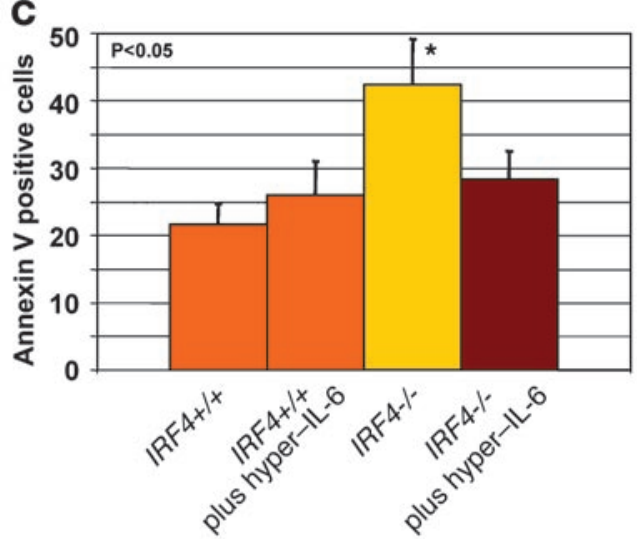

\section{Figure 7}

Hyper-IL-6 treatment induces IL-6 but not TNF production in IRF4-/- mice. (A) WT and IRF4-knockout mice were treated with TNBS, and some mice received hyper-IL-6. Relative expression levels of IL-6, TNF, and TGF- $\beta$ mRNA were measured by quantitative real-time RT-PCR on day 4. Values were normalized to $\beta$-actin expression levels. TNBS-treated IRF4-/- mice $(n=8)$ showed low mucosal expression of IL-6 mRNA. Hyper-IL-6 application induced a 14-fold increase of IL-6 production in TNBS-treated IRF4-/- mice $(n=6)\left({ }^{* \star} P<0.01\right)$. The expression levels of the proinflammatory cytokine TNF as well as levels of TGF- $\beta$ remained unaffected, however. Treatment of WT mice with hyper-IL-6 $(n=5$; WT mice, $n=4$ ) did not lead to a further significant increase of IL-6, TNF, or TGF- $\beta$. Data are shown as mean values \pm SEM from 3 experiments. (B and C) IRF4-knockout mice and WT mice were treated with TNBS, and the presence of apoptosis in gut mononuclear cells was determined by propidium iodide and annexin V staining using FACS analysis ( $n=6$ per group). IRF4-deficient mononuclear cells in the gut showed a significantly higher presence of cell apoptosis in TNBS colitis than in WT cells, and this could be abrogated by hyper-IL-6 administration. One representative experiment is shown. ${ }^{*} P<0.05$. (D) Apoptosis of gut mononuclear cells was determined by TUNEL assays. Hyper-IL-6 treatment prevented the induction of mononuclear cell apoptosis in the colon of IRF4-deficient mice. One representative experiment is shown $(n=4$ per group). Original magnification, $\times 300$.

than IL-17A control T cell-dependent colitis. Alternatively, other proinflammatory cytokines released by mucosal $\mathrm{T}$ cells in an IRF4dependent fashion might play a key functional role.

The cytokine IL- 6 is known to induce STAT- 3 expression in T cells and thereby control $\mathrm{T}$ cell resistance against apoptosis in experimental colitis (11-13). Here, we identified IRF4 as a key regulator of mucosal IL-6 production and T cell apoptosis in experimental colitis. The functional relevance of this finding was highlighted by the observation that treatment of IRF4-deficient mice with IL-6 or IL-6 plus the soluble IL-6R (hyper-IL-6) (30) abrogates the protective phenotype of these mice. Moreover, hyper-IL-6 treatment induced mucosal IL-6 production in IRF4-knockout mice and prevented mononuclear cell apoptosis in experimental colitis. Finally, IRF4-deficient T cells failed to induce mucosal IL- 6 production and colitis in adoptive transfer systems associated with an induction of mononuclear cell apoptosis. These data clearly suggest that the protective effects of IRF4 deficiency in T cell-dependent experimental colitis are due to reduced IL- 6 production with subsequent effects on mucosal cell apoptosis. As IL-6 plays a key role in inducing T cell resistance against apoptosis, thereby causing increased colitis activity (11), our data suggest that the reduced IL-6 levels produced in the absence of IRF 4 result in reduced T cell-dependent colitis activity by favoring mucosal $\mathrm{T}$ cell apoptosis.

Muramyl dipeptide has recently been shown to protect from dextran sodium sulfate colitis via IRF4 effects in APCs (31). In contrast, we demonstrate here a pathogenic role of IRF4 in T lymphocytes in experimental colitis. IRF4 thus has a dual function in experimental colitis. It should be noted, however, that the complete absence of IRF4 in both TNBS and oxazolone colitis had a marked protective function in experimental colitis, strongly suggesting that the regulatory effects of IRF4 on T cells play a predominant role in these models in vivo. The relevance of this observation for IBDs in humans is underlined by our finding that patients with active mucosal inflammation exhibit numerous IRF4-expressing $\mathrm{T}$ cells in the gut. Interestingly, such T cells in IBD are known to produce 
A

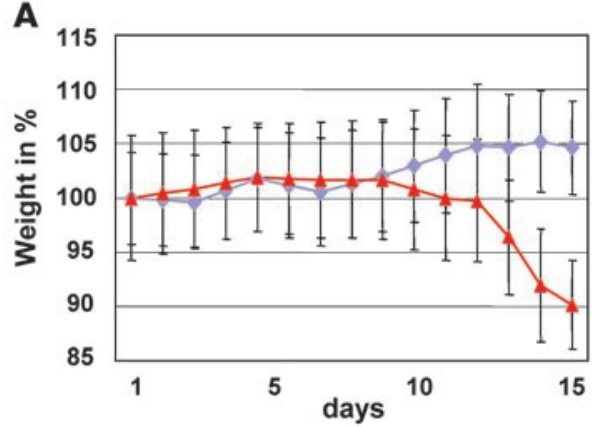

C

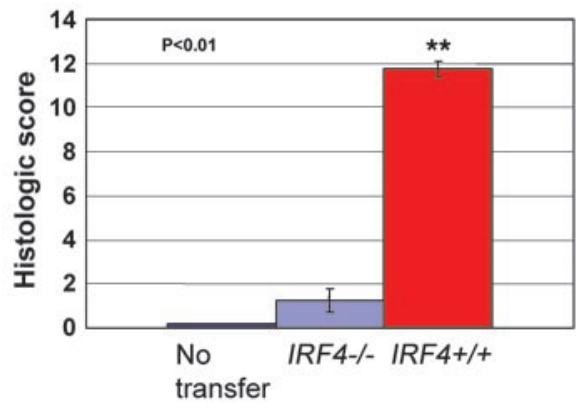

B

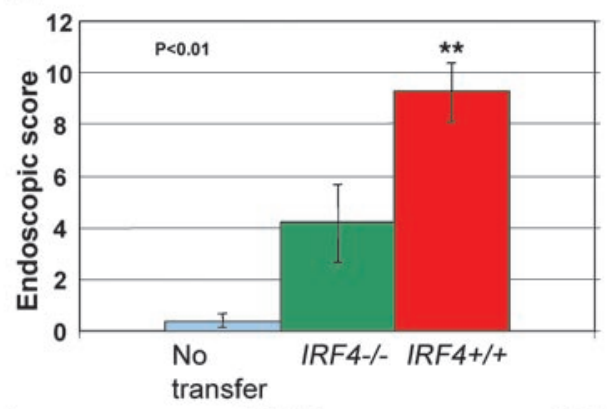

D

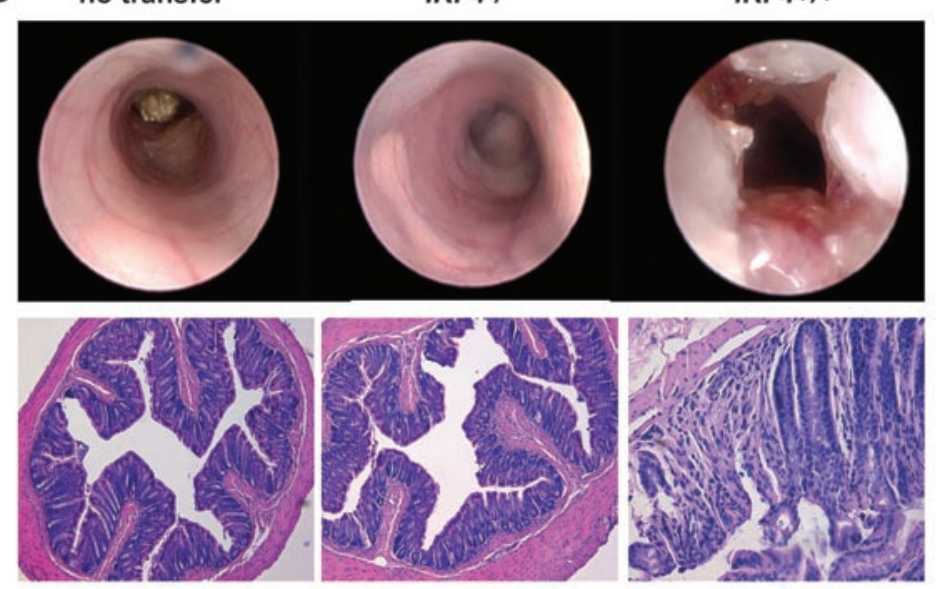

\section{Figure 8}

The protective effects of IRF4 deficiency are mediated via T lymphocytes: studies in the CD45RB hi adoptive transfer model of colitis. CD45RB ${ }^{\text {hiCD4 }} 4^{+}$T cells from WT or IRF4-/- mice were adoptively transferred into immunodeficient $R A G 2^{-/-}$mice. Whereas $R A G 2^{-/-}$mice reconstituted with WT T cells developed severe colitis accompanied by weight loss (A, red curve), mice given IRF4 ${ }^{-/-}$T cells were completely protected and even gained body weight (A, blue curve). One representative experiment out of 3 with 4 to 7 mice per group is shown. The differences between both groups were significant at days 14 and 15. (B) Endoscopy showed an inflamed mucosa in RAG2-knockout mice given WT T cells, whereas little or no inflammation was noted in $R A G 2^{-/-}$mice reconstituted with IRF4-deficient T cells. (C) Consistently, histological scoring ( $n=8$ per group) revealed significantly reduced colitis activity in the latter as compared with the former group of mice ( $\left.{ }^{\star *} P<0.01\right)$. Whereas the WT group showed marked inflammation, only a very mild colitis was noted in the IRF4-deficient group. (D) For each group, representative endoscopic and histologic pictures are shown. Original magnification, $\times 100$.

high amounts of IL-6 and are resistant against T cell apoptosis (11), consistent with the idea of a regulatory role of IRF4 in T cells in IBD. This concept is underlined by the observation that mucosal IRF4 levels in IBD correlate well with local IL-6 mRNA levels. In any case, our data identify what we believe is a novel role for IRF4 as a key regulator of mucosal immune homeostasis via $\mathrm{T}$ cell-derived IL-6 and highlight its potential as a therapeutic target in IBDs.

\section{Methods}

Human gut tissue. Colon samples were obtained from resection specimens from patients with $\mathrm{CD}(n=10)$ (colonic disease) and UC $(n=10)$. Surgery was performed because of stenoses, fistulas, or therapy-refractory disease. The CD group consisted of 4 male and 6 female patients between 20 and 50 years. At the time of surgery, patients were treated with either 5-ASA, corticosteroids, or anti-TNF antibodies. Five patients were receiving azathioprine. The UC group consisted of 5 male and 5 female patients, 25-60 years of age. At the time of resection, all patients were receiving corticosteroids and 4 were receiving azathioprine. The control group consisted of 10 patients (5 male, 5 female; age, 30-60 years) not receiving corticosteroids or immunosuppressants.
For real-time PCR, mRNA from endoscopic biopsy specimens of 10 control patients and 16 IBD patients was used. Groups were age matched. Among the IBD patients, 10 suffered from CD and 6 from UC. Treatment consisted of systemic corticosteroids, salicylates, and budesonide.

The collection of surgical samples and biopsies was approved by the ethical committee and the institutional review board of the University of Mainz, and each patient gave written informed consent.

Immunofluorescence staining. Mucosal samples were snap-frozen and embedded in OCT compound to obtain cryosections. Immunofluorescence was done on gut cryosections. Tissues were fixed in 4\% paraformaldehyde in PBS and washed in $0.01 \mathrm{M}$ PBS. Samples were then pretreated with $10 \%$ serum in a solution of $0.1 \%$ Triton X-100 in PBS and incubated overnight at $4{ }^{\circ} \mathrm{C}$ with primary antibody (polyclonal rabbit antibody against IRF4; Santa Cruz Biotechnology Inc.) in $0.3 \%$ bovine serum albumin and $0.1 \%$ Triton X-100 in PBS. Samples without primary antibody served as negative control. The next day, samples were rinsed in PBS and incubated with a biotinylated secondary IgG antibody (1:100-1:200 dilution; Vector Laboratories) followed by incubation with streptavidine-conjugated Cy2 or Cy3 (1:500-1:1,000 dilution; all from Dianova). Samples were subjected to a second cycle of staining by using monoclonal mouse antibodies against human CD3, CD4, 
A

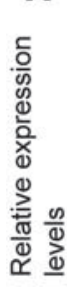

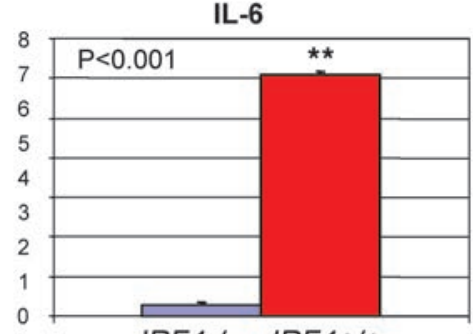

IRF4-/- IRF4+/+

TGF-beta

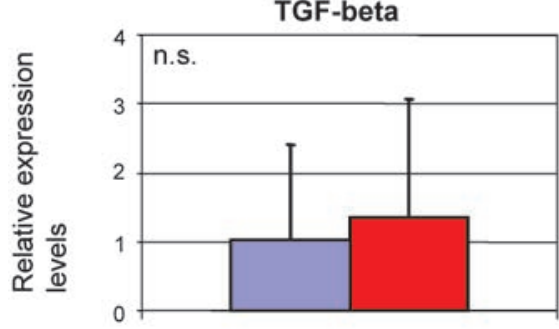

TNF

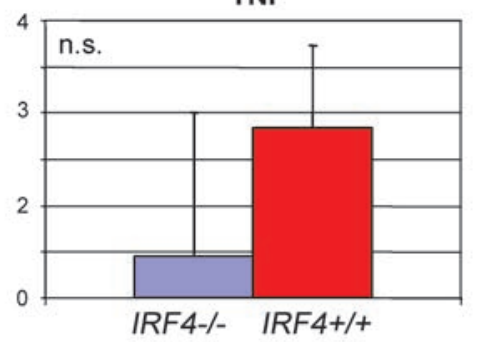

B

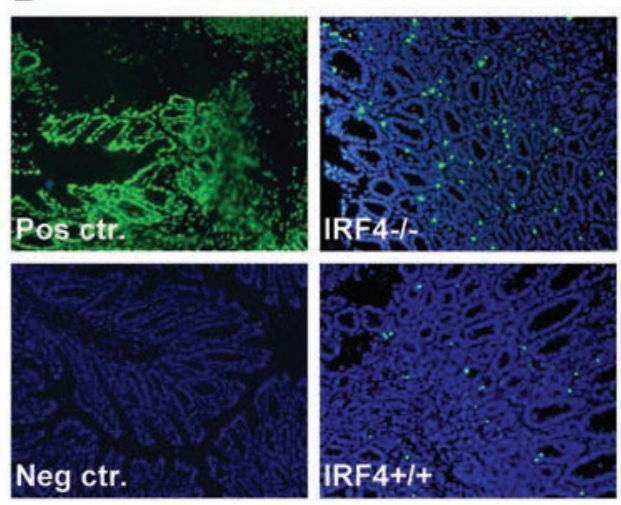

Figure 9

Analysis of cytokine expression and apoptosis in the colon of reconstituted mice. (A) Expression of cytokine mRNA levels in the colon of RAG2knockout mice reconstituted with WT or IRF4-deficient T cells was performed by quantitative PCR. Whereas mucosal IL-6 expression was significantly higher $\left({ }^{\star \star} P<0.01\right)$ in RAG2-knockout mice reconstituted with WT but not IRF4-deficient cells, no significant differences were noted in TNF and TGF- $\beta$ levels. One representative experiment out of 3 is shown. (B) In addition, TUNEL assays on colonic cryosections were performed to detect apoptotic LPMCs. There was a significantly higher number of apoptotic LPMCs in the colons of mice reconstituted with IRF4-deficient T cells as compared with mice given WT T cells. Original magnification, $\times 200$.

$\mathrm{CD} 8$, and CD11c as primary antibodies (all obtained from BD Biosciences - Pharmingen) and streptavidine-conjugated Cy3 as a chromogen. Slides were mounted with mounting medium for fluorescence (Vector Laboratories) and analyzed with an Olympus microscope. Finally, cells in 7 highpower fields (HPFs) were counted in all patients for each condition.

To detect IRF4 and IL-6 in murine colon samples, cryosections were fixed in ice-cold acetone for 10 minutes followed by sequential incubation with methanol, avidin/biotin (Vector Laboratories), and protein-blocking reagent (Dako) to eliminate unspecific background staining. Slides were then incubated overnight with primary antibodies directed against IRF4 and IL-6 (all from Santa Cruz Biotechnology Inc.). Subsequently, the slides were incubated for 30 minutes at room temperature with biotinylated secondary antibodies (Dianova). All samples were finally treated with streptavidine-conjugated $\mathrm{Cy} 3$. Before examination, the nuclei were counterstained with Hoechst 3342 (Molecular Probes).

Animals and induction of colitis. The TNBS and oxazolone colitis models have been well established in the laboratory as previously described $(32,33)$. Transfer colitis was performed as described by Morrissey and Powrie $(34,35)$. In brief, $\mathrm{CD} 4{ }^{+} \mathrm{CD} 45 \mathrm{RB}^{\text {hi }} \mathrm{T}$ cells were sorted by flow cytometry (FACSCalibur; $\mathrm{BD})$, and $5 \times 10^{5}$ cells were intraperitoneally transferred into Rag2 ${ }^{-/-}$mice. $\mathrm{T}$ cells were obtained either from $I R F 4^{-/-}$or $\mathrm{C} 57 \mathrm{BL} / 6$ donors, as specified below. For modulation of colitis activity, mice were given $1 \mu \mathrm{g}$ hyper-IL- 6 or $5 \mu \mathrm{g}$ IL- 6 by intraperitoneal injection. Injection was made 7 days after skin sensitization with TNBS or oxazolone just before intrarectal administration of the hapten reagents. All animal studies were approved by the Institutional Animal Care and Use Committee of the University of Mainz.

For chronic oxazolone-induced colitis, 3 intrarectal administrations of oxazolone were performed at days 7, 12, and 17 after initial sensitization to oxazolone. Final evaluation was performed 21 days after sensitization.

Preparation of gut tissues. Sections of distal and proximal colon were snapfrozen and either homogenized or embedded in OCT compound to obtain cryo sections. Paraffin-embedded sections were stained with H\&E for his- tological scoring of colitis severity. In transfer colitis, the murine colon was divided into a proximal and distal segment and 1 piece of each segment was used to isolate mRNA.

Histologic analysis of colon cross sections and endoscopy. Tissues were removed from colitic mice, formalin fixed, and embedded in paraffin, and sections were made and stained with H\&E. The degree of inflammation on microscopic cross sections of the colon was graded semiquantitatively on a scale of $0-6$ or alternatively of $0-14$ in the case of transfer colitis.

Histological scoring was performed in a blinded fashion by pathologists (H.A. Lehr and S. Biesterfeld). A combined score ranging from 0 to 6 was used to screen TNBS and oxazolone colitis. Inflammatory cell infiltration was scored from $0-3$, and tissue damage was scored from $0-3$. Occasional or no inflammatory infiltrate in the lamina propria was scored as 0 , increased numbers of inflammatory cells restricted to the lamina propria were scored as 1 , inflammatory infiltrates reaching the submucosa were scored as 2 , and transmural inflammation was scored as 3 . The subscore for tissue damage took into account epithelial lesions: no mucosal damage was scored as 0 , focal crypt lesions were scored as 1 , surface mucosal erosions or focal ulcerations were scored as 2 , and extensive mucosal damage affecting the submucosa was scored as 3 . The combined inflammatory and histological score resulted in the overall score ranging from 0 (no changes) to 6 (severe inflammatory infiltrate and mucosal damage).

To assess the histopathological alterations of the colon in the CD4 T cell transfer model, a scoring system ranging from $0-14$ was used and the pathologist (C. Mueller) was blinded to sample identity. The following parameters were included: (a) mucin depletion/loss of goblet cells (scores from 0 to 3); (b) crypt abscesses (scores from 0 to 3); (c) epithelial erosions (scores from 0 to 1); (d) hyperemia (scores from 0 to 2); (e) cellular infiltration (scores from 0 to 3 ); and (f) thickness of colonic mucosa (scores from 0 to 2 ). These individual scores were added to obtain the final histopathology score for each sample (score 0 , no alterations; score 14 , most severe signs of colitis). 
To perform experimental endoscopy, mice were anesthetized using intraperitoneal injection of Avertine (Sigma-Aldrich). Mouse endoscopy was performed using a miniature endoscope (scope $1.9 \mathrm{~mm}$ outer diameter), a xenon light source, a triple-chip camera, and an air pump (all from Karl Storz), and MEICS score was used for assessment as previously described (36).

Cell isolation and purification of spleen mononuclear cells. Spleen mononuclear cells were isolated from freshly obtained spleen specimens from healthy B6 mice. Erythrocytes were removed from spleen cell suspension by hypotonic lysis in ammonium chloride and potassium chloride (ACK) buffer. The MACS system (Miltenyi Biotec) was used to isolate CD4 ${ }^{+} \mathrm{T}$ cells (purity $>95 \%$, as determined by FACS analysis). The cells were cultured at a density of $1 \times 10^{6} / \mathrm{ml}$ in complete RPMI medium in a humidified atmosphere with $5 \% \mathrm{CO}_{2}$ in a $37^{\circ} \mathrm{C}$ incubator in the presence or absence of antibodies against mouse CD3 and CD28 (CD3, clone 145-2C11, $2 \mu \mathrm{g} / \mathrm{ml}$; CD28, clone 37.51, $0.5 \mu \mathrm{g} / \mathrm{ml}$; BD Biosciences - Pharmingen).

Cell isolation and purification of LPMCs. LPMCs were isolated from freshly obtained murine colon specimens using a modification of the technique described by van der Heijden and Stok (37). After removal of Peyer patches, the colon was washed in HBSS free of calcium and magnesium and incubated twice in HBSS containing $5 \mathrm{mM}$ EDTA for 15 minutes at $37^{\circ} \mathrm{C}$. After incubation, the epithelial cell layer and intraepithelial lymphocytes were removed by intensive vortexing and passing through a $100-\mu \mathrm{m}$ cell strainer; new EDTA solution was added. Next, the tissue was washed in HBSS, cut in $1-\mathrm{mm}^{2}$ pieces, and placed in $5 \mathrm{ml}$ digestion solution containing $4 \%$ fetal calf serum, $0.5 \mathrm{mg} / \mathrm{ml}$ collagenase D, DNase I grade 2 , and $50 \mathrm{U} / \mathrm{ml}$ dispase II (all obtained from Roche). Digestion was performed by incubating the pieces at $37^{\circ} \mathrm{C}$ for 20 minutes. After the initial 20 minutes, the solution was passed through a $100-\mu \mathrm{m}$ cell strainer, and the pieces were collected and placed into fresh digestion solution 3 times. Supernatants were washed in cold FACS buffer, resuspended in $10 \mathrm{ml}$ of the $40 \%$ fraction of a 40:80 Percoll gradient, and overlaid on $5 \mathrm{ml}$ of the $80 \%$ fraction in a $15-\mathrm{ml}$ tube. Lamina propria (LP) cells were collected at the interphase of the Percoll gradient, washed once, and resuspended in FACS buffer or cell culture medium at a density of $10^{6}$ cells per $\mathrm{ml}$. In some experiments, the MACS system (Miltenyi Biotec) was subsequently used to isolate $\mathrm{CD} 90^{+} \mathrm{T}$ cells from the lamina propria (purity $>90 \%$, as determined by FACS analysis).

Cytokine measurement. Cytokine concentration was measured in supernatants of mononuclear spleen cells or isolated splenic $\mathrm{CD} 4^{+} \mathrm{T}$ cells, LPMCs, and $\mathrm{CD} 90^{+}$lamina propria $\mathrm{T}$ cells. Quantification was performed using multiple cytokine measurement kits and flow cytometry or sandwichELISA according to the manufacturer's instructions (Bender MedSystems). Cells were cultured and stimulated for 24 or 48 hours using anti-CD 3 and anti-CD28 antibodies (CD3, clone 145-2C11, $2 \mu \mathrm{g} / \mathrm{ml}$; CD28, clone 37.51, $0.5 \mu \mathrm{g} / \mathrm{ml}$; BD Biosciences - Pharmingen).

Detection of apoptosis. Spleen mononuclear cells and LPMCs were isolated as described above. The cells were cultured at a density of $1 \times 10^{6} / \mathrm{ml}$ in complete RPMI medium in a humidified atmosphere with $5 \% \mathrm{CO}_{2}$ in a $37^{\circ} \mathrm{C}$ incubator in the presence of antibodies against mouse $\mathrm{CD} 3$ and CD28 (CD3, clone 145-2C11, $2 \mu \mathrm{g} / \mathrm{ml}$; CD28, clone 37.51, $0.5 \mu \mathrm{g} / \mathrm{ml}$; BD Biosciences - Pharmingen). Cells were cultured in the presence or absence of hyper-IL-6. For FACS analysis, apoptotic cells were detected by staining with annexin $V$ and propidium iodide using the Annexin V FITC Apoptosis Detection Kit I (BD Biosciences - Pharmingen). In addition, double staining of spleen cells and LPMCs was performed using annexin V (FITC) and anti-CD3 antibodies (PE conjugated), as previously described (10-13).
To visualize apoptotic cells on colonic cryosections, TUNEL reaction was performed using ApoTag Fluorescein Kit (Appligene Oncor), according to the manufacturer's instructions. Following washing, the cells were counterstained with the DNA binding dye DAPI $(1 \mu \mathrm{g} / \mathrm{ml})$ for visualization of nuclei and mounted in VECTASHIELD (Vector Laboratories) for fluorescence microscopy.

Real-time PCR. Total RNA from murine colon specimens was extracted using RNeasy Mini Kit (QIAGEN). $1 \mu \mathrm{g}$ of total RNA was reverse transcribed using an oligo-dT primer. Real-time PCR analysis for quantification of expression of IL- 6 , TNF, TGF- $\beta$, IL-4, IFN- $\gamma$, and $\beta$-actin mRNAs was performed in duplicate on an iCycler (Bio-Rad Laboratories) using the ABsolute SYBR Green Fluorescein Kit (Abgene; Thermo Scientific). QuantiTect primer sets were used for these experiments (QIAGEN).

The relative increase in reporter fluorescent dye emission was monitored. The level of cytokine mRNA relative to actin was calculated using the following formula: relative cytokine mRNA expression $=$ $2^{\wedge}\left[c_{t}\left(\right.\right.$ cytokine $\left._{\text {control }}\right)-c_{t}\left(\right.$ cytokine $\left._{\text {treated }}\right)+c_{t}\left(\right.$ actin $\left._{\text {treated }}\right)-c_{t}\left(\right.$ actin $\left.\left._{\text {control }}\right)\right]$, where $c_{t}$ is defined as the number of the cycle in which emission exceeds an arbitrarily defined threshold.

Total RNA from human colon biopsy was extracted using RNeasy Mini Kit (QIAGEN). $1 \mu \mathrm{g}$ of total RNA was reverse transcribed using random hexamer primer (Amersham). Real-time PCR analysis for quantification of expression of IRF4, IL-6, and GAPDH mRNAs was performed in duplicate on a light cycler (Roche) using a QIAGEN SYBR Green Kit and QuantiTect primer sets (QIAGEN). Using GAPDH as a reference, the relative expression level of cytokine mRNA was calculated with the following formula: relative cytokine mRNA expression $=2^{\wedge}\left[c_{t}\left(\right.\right.$ cytokine $\left._{\text {control }}\right)$ $-c_{t}($ cytokine treated $\left.)+c_{t}\left(G A P D H_{\text {treated }}\right)-c_{t}\left(G A P D H_{\text {control }}\right)\right]$, where $c_{t}$ is defined as the number of the cycle in which emission exceeds an arbitrarily defined threshold.

Statistics. Statistical significance of evaluated data was tested using MannWhitney $U$ Test and Student's $t$ test. Results were considered as statistically significant at $P<0.05$. Results are expressed as mean values $( \pm$ SD or \pm $\mathrm{SEM})$. The error bars in histogram figures represent SD or SEM as indicated. Relative expression of IRF4 and IL- 6 mRNA from each patient was correlated by using the linear correlation coefficient $r$ to measure the strength and the direction of a linear relationship between these 2 variables.

\section{Acknowledgments}

The work from M.F. Neurath and J. Mudter was supported by a grant from the Stiftung Rheinland-Pfalz für Innovation. M. Lohoff was supported by the DFG grant LO396-1. M.F. Neurath was supported by grants from the SFB548 of the DFG. The technical assistance of Francoise Burri is gratefully acknowledged. The authors thank Katrin Rücknagel for excellent support and technical assistance.

Received for publication July 10, 2007, and accepted in revised form April 30, 2008.

Address correspondence to: Markus F. Neurath, 1st Department of Internal Medicine and Institute of Molecular Medicine, University of Mainz, Langenbeckstrasse 1, 55131 Mainz, Germany. Phone: 49-6131-175740; Fax: 49-6131-175583; E-mail: neurath@1-med. klinik.uni-mainz.de.

\footnotetext{
1. Macdonald, T.T., and Monteleone, G. 2005. Immunity, inflammation, and allergy in the gut. Science. 307:1920-1925.

2. Podolsky, D.K. 2002. Inflammatory bowel disease. N. Engl. J. Med. 347:417-429.
}

3. Sartor, R.B. 2004. Therapeutic manipulation of the enteric microflora in inflammatory bowel diseases: antibiotics, probiotics, and prebiotics. Gastroenterology. 126:1620-1633.

4. Shanahan, F. 2002. Crohn's disease. Lancet.
359:62-69.

5. Wehkamp, J., et al. 2005. Defensin deficiency, intestinal microbes, and the clinical phenotypes of Crohn's disease. J. Leukoc. Biol. 77:460-465.

6. Mudter, J., and Neurath, M.F. 2003. Mucosal T-cells: 
mediators or guardians of inflammatory bowel disease? Curr. Opin. Gastroenterol. 19:343-349.

7. Strober, W., Fuss, I.J., and Blumberg, R.S. 2002. The immunology of mucosal models of inflammation. Annu. Rev. Immunol. 20:495-549.

8. Blumberg, R.S., Saubermann, L.J., and Strober, W. 1999. Animal models of mucosal inflammation and their relation to human inflammatory bowel disease. Curr. Opin. Immunol. 11:648-656.

9. Fuss, I.J., et al. 1996. Disparate CD4+ lamina propria (LP) lymphokine secretion profiles in inflam matory bowel disease. Crohn's disease LP cells manifest increased secretion of IFN-gamma, whereas ulcerative colitis LP cells manifest increased secretion of IL-5. J. Immunol. 157:1261-1270.

10. Mudter, J., et al. 2002. A new model of chronic colitis in SCID mice induced by adoptive transfer of CD62L+CD4+ T Cells: insights into the regulatory role of interleukin-6 on apoptosis. Pathobiology. 70:170-176.

11. Atreya, R., et al. 2000. Blockade of interleukin 6 trans signaling suppresses T-cell resistance against apoptosis in chronic intestinal inflammation: evidence in Crohn disease and experimental colitis in vivo. Nat. Med. 6:583-588.

12. Yamamoto, M., et al. 2000. IL-6 is required for the development of Th1 cell-mediated murine colitis. J. Immunol. 164:4878-4882.

13. Kai, Y., et al. 2005. Colitis in mice lacking the common cytokine receptor gamma chain is mediated by IL-6-producing CD4+ T cells. Gastroenterology. 128:922-934.

14. Ito, H., et al. 2004. A pilot randomized trial of a human anti-interleukin- 6 receptor monoclonal antibody in active Crohn's disease. Gastroenterology. 126:989-996.

15. Mittrucker, H.W., et al. 1997. Requirement for the transcription factor LSIRF/IRF4 for mature B and T lymphocyte function. Science. 275:540-543.

16. Lohoff, M., et al. 2002. Dysregulated T helper cell differentiation in the absence of interferon regulatory factor 4. Proc. Natl. Acad. Sci. U. S. A. 99:11808-11812.

17. Brass, A.L., Zhu, A.Q., and Singh, H. 1999. Assembly requirements of PU.1-Pip (IRF-4) activator complexes: inhibiting function in vivo using fused dimers. EMBO J. 18:977-991.

18. Brass, A.L., et al. 1996. Pip, a lymphoid-restricted IRF, contains a regulatory domain that is important for autoinhibition and ternary complex formation with the Ets factor PU.1. Genes Dev. 10:2335-2347.

19. Tominaga, N., et al. 2003. Development of Th1 and not Th2 immune responses in mice lacking IFNregulatory factor-4. Int. Immunol. 15:1-10.

20. Rengarajan, J., et al. 2002. Interferon regulatory factor 4 (IRF4) interacts with NFATc2 to modulate interleukin 4 gene expression. J. Exp. Med. 195:1003-1012.

21. Lohoff, M., and Mak, T.W. 2005. Roles of interferonregulatory factors in T-helper-cell differentiation. Nat. Rev. Immunol. 5:125-135.

22. Tamura, T., et al. 2005. IFN regulatory factor-4 and 8 govern dendritic cell subset development and their functional diversity. J. Immunol. 174:2573-2581.

23. Honma, K., et al. 2005. Interferon regulatory factor 4 negatively regulates the production of proinflammatory cytokines by macrophages in response to LPS. Proc. Natl. Acad. Sci. U. S. A. 102:16001-16006

24. van der Stoep, N., et al. 2004. E47, IRF-4, and PU.1 synergize to induce B-cell-specific activation of the class II transactivator promoter III (CIITA-PIII). Blood. 104:2849-2857.

25. Brüstle, A., et al. 2007. The development of inflammatory $\mathrm{T}(\mathrm{H})-17$ cells requires interferon-regulatory factor 4. Nat. Immunol. 8:958-966.

26. Yen, D., et al. 2006. IL-23 is essential for T cellmediated colitis and promotes inflammation via IL-17 and IL-6. J. Clin. Invest. 116:1310-1316.

27. Fuss, I.J., et al. 2006. Both IL-12p70 and IL-23 are synthesized during active Crohn's disease and are down-regulated by treatment with anti-IL-12 p40 monoclonal antibody. Inflamm. Bowel Dis. 12:9-15.

28. Tato, C.M., Laurence, A., and O'Shea, J.J. 2006. Helper T cell differentiation enters a new era: le roi est mort; vive le roi! J. Exp. Med. 203:809-812.

29. Ivanov, I.I., et al. 2006. The orphan nuclear receptor RORgammat directs the differentiation program of proinflammatory IL-17+ T helper cells. Cell. 126:1121-1133.

30. Peters, M., et al. 1998. In vivo and in vitro activities of the gp130-stimulating designer cytokine HyperIL-6. J. Immunol. 161:3575-3581.

31. Watanabe, T., et al. 2008. Muramyl dipeptide activation of nucleotide-binding oligomerization domain 2 protects mice from experimental colitis. J. Clin. Invest. 118:545-559.

32. Boirivant, M., et al. 1998. Oxazolone colitis: A murine model of $\mathrm{T}$ helper cell type 2 colitis treatable with antibodies to interleukin 4. J. Exp. Med. 188:1929-1939.

33. Neurath, M.F., et al. 1995. Antibodies to interleukin 12 abrogate established experimental colitis in mice. J. Exp. Med. 182:1281-1290.

34. Morrissey, P.J., et al. 1993. CD4+ T cells that express high levels of CD45RB induce wasting disease when transferred into congenic severe combined immunodeficient mice. Disease development is prevented by cotransfer of purified CD 4+ T cells. J. Exp. Med. 178:237-244.

35. Powrie, F., et al. 1993. Phenotypically distinct subsets of CD4+ $\mathrm{T}$ cells induce or protect from chronic intestinal inflammation in C. B-17 scid mice. Int. Immunol. 5:1461-1471.

36. Wirtz, S., et al. 2002. Treatment of T cell-dependent experimental colitis in SCID mice by local administration of an adenovirus expressing IL-18 antisense mRNA. J. Immunol. 168:411-420.

37. Van der Heijden, P.J., and Stok, W. 1987. Improved procedure for the isolation of functionally active lymphoid cells from the murine intestine. J. Immunol. Methods. 103:161-167. 